\title{
MS25-P03 | AUTOMATED ELECTRON DIFFRACTION: 3D STRUCTURE DETERMINATION WITH SUB-ÅNGSTRÖM RESOLUTION
}

Roslova, Maria (Stockholm University, Stockholm, SWE); Smeets, Stef (Delft University of Technology, Delft); Wang, Bin (Stockholm University, Stockholm, SWE); Thersleff, Thomas (Stockholm University, Stockholm, SWE); Hu, Hongyi (Stockholm University, Stockholm, SWE); Zou, Xiaodong (Stockholm University, Stockholm, SWE)

Electron diffraction (ED) is a well-known technique for 3D structure determination of solids, which is especially advantageous for the study of micro- and nanocrystals. Several software-based methods for automated collection of 3D ED data have been developed, however, until now many automation packages were optimized for specific microscopes available in the labs that created the software. A new custom DigitalMicrigraph (DM) script InsteaDMatic has been developed to facilitate rapid semi-automated electron diffraction data acquisition on our Themis Z microscope with a Gatan One View CCD camera. The InsteaDMatic communicates to both the microscope and camera via DM interfaces, and thus can in principle be directly used in all microscopes with a Gatan CCD camera, where the hardware communications are by default already set up by the DM software.

Benefiting from InsteaDMatic, we collected high-quality data on randomly-oriented crystals of ZSM-5 zeolite down to $50-100 \mathrm{~nm}$ in size. The method delivers up to $0.8 \AA$ resolution data, suitable for "ab-initio" crystal structure analysis by XDS and SHELX-97 software. Positions of the Si and O atoms in ZSM-5 can be determined within an accuracy of more than $0.1 \AA$. Thus, the development of the cross-platform software makes cRED with a subångström resolution available for all microscopes utilizing the DM software. 